\title{
Prospects for Gallium Nitride-on-Diamond Transistors
}

\author{
J.D. Blevins, G.D.Via \\ Air Force Research Laboratory (AFRL), Wright-Patterson AFB, Ohio, 45433, USA
}

\begin{abstract}
Strategies aimed at improving the near junction heat removal of Gallium Nitride (GaN) High Electron Mobility Transistors (HEMTs) are presently limiting GaN device technology from realization of its true capability [1]. Approximately ten years ago, Cree demonstrated AlGaN/GaN HEMTs with power densities exceeding $40 \mathrm{~W} / \mathrm{mm}$ [2]. Control of the GaN junction temperature requires integration of thermal transport solutions near the heat source to ensure optimal performance and reliable operation [3]. An approach under consideration is the use of Chemical Vapor Deposition (CVD) polycrystalline diamond inserted within microns of the device junction. Recent AFRL and Defense Advanced Research Projects Agency (DARPA) efforts have shown that replacing the epitaxial host substrate with high thermal conductivity polycrystalline diamond substrates can improve the GaN HEMT areal power density $>3$ Xs [4-9]. This paper will examine the motivation behind the use of diamond, integration approaches, material/device results and key technological challenges going forward.
\end{abstract}

Index Terms - GaN, HEMT, CVD polycrystalline diamond, thermal management.

\section{INTRODUCTION}

GaN power amplifiers are rapidly becoming the technology of choice for a variety of military radar, electronic warfare and communication systems as well as commercial wireless infrastructure components. Significant advances in epitaxial growth, device fabrication and design have enhanced the electrical performance to a point where thermal management is now a primary limiting factor in device and circuit operation [10].

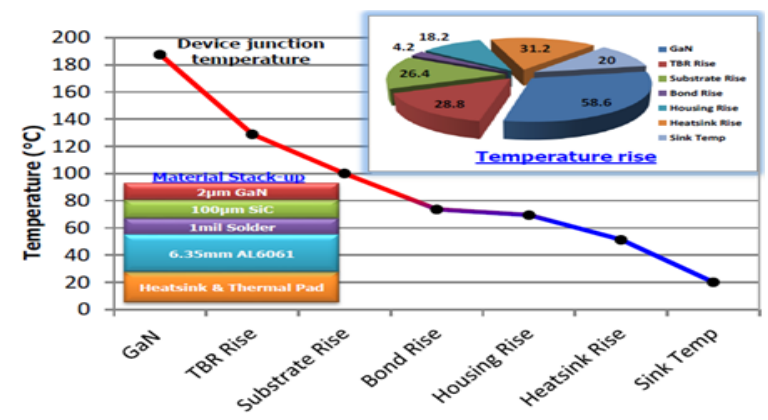

Fig. 1. Conventional GaN-on-SiC HEMT stack-up [10].
Silicon, silicon carbide $(\mathrm{SiC})$ sapphire and $\mathrm{GaN}$ are commercially available to support $\mathrm{GaN}$ device fabrication. However, even use of $\mathrm{SiC}$ substrates, with a thermal conductivity of $400 \mathrm{~W} / \mathrm{m}-\mathrm{K}$, cannot effectively manage device self-heating. A typical GaN-on-SiC HEMT thermal profile is shown in Fig. 1. The GaN epitaxy, interfaces and $\mathrm{SiC}$ substrate dominate the overall temperature rise. In order to mitigate thermally-induced performance and reliability degradation, useable power densities are limited to 5-8 W/mm [5].

Military electronics reliance on "remote" cooling solutions has resulted in thermal management hardware accounting for a large fraction of the SWaPC of RF components [11]. Overcoming the remote cooling paradigm requires reducing the thermal resistance of the near-junction region and heat sink without producing deleterious effects in electrical performance [12]. The continued use of conventional thermal conduction and spreading techniques combined with remote cooling is limiting the realization of GaN's intrinsic electronic capabilities.

Improved thermal transport solutions capable of managing localized device hot spots are required to obtain peak performance and reliable operation. Replacing $\mathrm{SiC}$ with a higher thermal conductivity substrate could substantially improve heat spreader near the hot spot lowering the overall temperature rise. The most promising approaches under development involve the integration of high thermal conductivity CVD polycrystalline diamond in close proximity of the device hot spots. The electrically insulating and thermal conductivity properties of CVD polycrystalline diamond provide an attractive option. Recent demonstrations of diamond integrated within microns of the $\mathrm{GaN}$ transistor junction has proven to be effective at reducing intra-device thermal resistance enabling significant increases in HEMT areal power density $\left(\mathrm{W} / \mathrm{mm}^{2}\right)$ without commensurate increases in operating temperature [12]. The decreased thermal resistance between the heat sources and diamond reduces the thermal constraints providing the device designer additional options to exploit the inherent characteristics of $\mathrm{GaN}$ 
[11]. Since the device operates cooler, it is possible to reduce the device gate pitch to achieve higher areal output power while maintaining an acceptable junction temperature. BAE has shown the gate pitch of a GaN-on-Diamond HEMT can be reduced from $49 \mu \mathrm{m}$ to $10 \mu \mathrm{m}$, enabling a $>5 \mathrm{X}$ improvement in power over GaN-on-SiC HEMT [3].

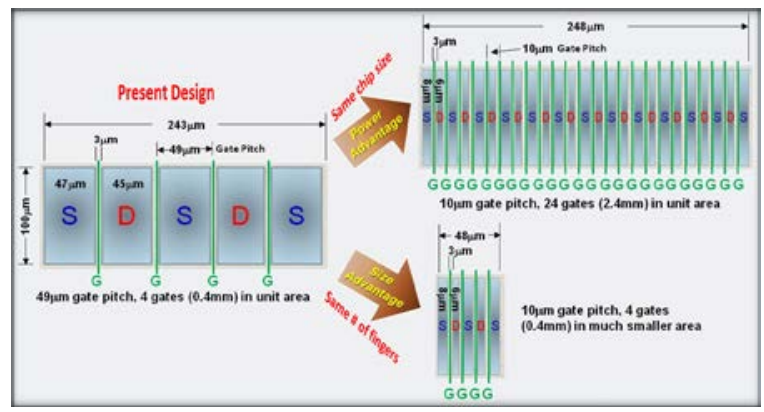

Fig. 2. The use of embedded polycrystalline diamond provides flexibility to reduce gate pitch to increase device periphery or reduce device area [3].

\section{DIAMOND PROCESS DESCRIPTIONS}

Since 2012, DARPA's Near Junction Thermal Transport (NJTT) Program performers have focused on developing an integrated embedded cooling approach using high thermal conductivity polycrystalline diamond [9]. NJTT Program demonstrations of GaN-on-Diamond HEMTs have proven to be effective at reducing intra-device thermal resistance enabling $>3 \mathrm{X}$ 's increase in $\mathrm{GaN}$ HEMT areal power density $\left(\mathrm{W} / \mathrm{mm}^{2}\right)$ without commensurate increases in operating temperature [9]. Presently, there are three primary GaN-on-Diamond fabrication processes under development. Notwithstanding the process differences and difficulties, each has shown performance benefits.

\section{A. Direct Growth of CVD Diamond}

This process deposits a CVD film of polycrystalline diamond on the N-face of a $\mathrm{GaN}$ HEMT epi wafer. Use of this material led to the first demonstration of a GaN-on-Diamond transistor by AFRL in 2007 [4]. The process flow is shown in Fig. 3. It begins with $\mathrm{AlGaN} / \mathrm{GaN}$ epitaxial layers grown on silicon (111) substrates. A sacrificial silicon handle wafer is attached to the front-side of the epi wafer to facilitate removal of the silicon host substrate. The silicon substrate and epitaxial transition layers are subsequently removed and a nominal $30 \mathrm{~nm}$ dielectric layer is deposited on the GaN buffer backside to facilitate microwave plasma CVD polycrystalline diamond growth. The addition of the dielectric layer does introduce a thermal boundary resistance (TBR) that must be minimized. Growth of approximately $100 \mu \mathrm{m}$ of polycrystalline diamond provides the necessary mechanical and thermal robustness for subsequent front side device fabrication as well as being compatible with microstrip MMIC backside processing requirements. The handle wafer is removed and the GaN-on-Diamond wafer is ready for standard front side processing. Prolonged high temperature exposure during CVD polycrystalline diamond growth does induce wafer strain resulting in significant wafer bow. This bow must be carefully managed to facilitate wafer scale processing. Several groups, including NJTT Program performers Raytheon and Qorvo (RFMD/ Triquint), along with AFRL have demonstrated that the diamond integration process did not significantly degrade the electrical performance and clear trends show improved thermal performance utilizing variants of this diamond integration approach [4-13].

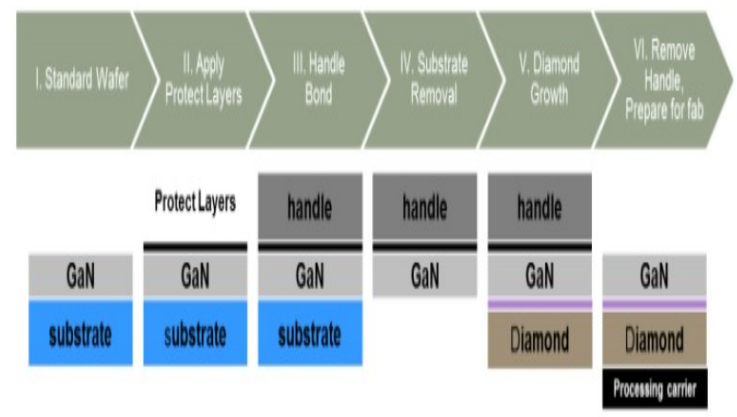

Fig. 3. Direct diamond growth process flow, $75 \mathrm{~mm}$ GaN-onDiamond device wafer and example of HEMT area reduction due to compaction of gate to gate spacing [15].

\section{B. Wafer Bonding}

BAE is developing a diamond wafer bonding process as shown in Fig. 4 that takes full advantage of their standard 100mm GaN-on-SiC MMIC fabrication process. This process utilizes a "device first" process that leverages a mature, reproducible GaN-on-SiC MMIC process. Following front side completion of a $100 \mathrm{~mm}$ GaN-on-SiC wafer, a silicon carrier wafer is bonded to the front side facilitate $\mathrm{SiC}$ substrate removal. A plasma etch is used to remove the $\mathrm{SiC}$ substrate and expose the backside $\mathrm{GaN}$ epi layer. The $\mathrm{GaN}$ epitaxial layer is further etched and chemicalmechanical polished (CMP) to a target of $<1 \mathrm{~nm}$ RMS surface roughness. A thin $\mathrm{SiN}$ bonding layer is deposited on the GaN buffer and polycrystalline diamond surfaces to facilitate low temperature atomic attachment. 


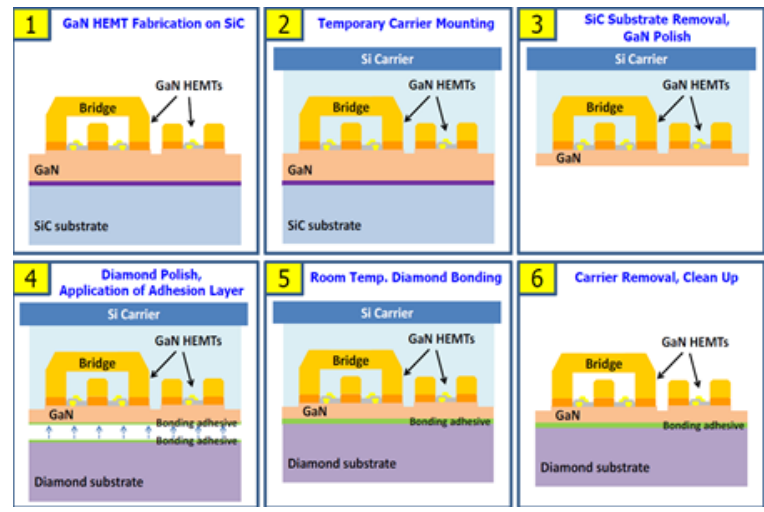

Fig. 4. Process flow for wafer bonding GaN device layers to a free-standing polycrystalline diamond substrate [10].

\section{Selective Polycrystalline Diamond Growth}

Northrop Grumman Aerospace Systems (NGAS) is developing an approach that selectively deposits CVD polycrystalline diamond in "thermal" via's located under device hot spots. This approach is fully compatible with existing GaN-on-SiC MMIC manufacturing processes. A cross section of a $\mathrm{GaN}-$ on-Diamond HEMT with large thermal via's filled with polycrystalline diamond is shown in Fig. 5. This approach deposits CVD polycrystalline diamond in areas requiring improved thermal spreading rather than covering the entire $\mathrm{SiC}$ backside. As such wafer stress resulting from CVD polycrystalline diamond growth is reduced. Thermal via's are formed using a high selectivity $\mathrm{SiC}$ dry etch that uses the AlN epilayer in the $\mathrm{GaN}$ buffer as an etch stop. Microwave plasma CVD polycrystalline diamond is grown in the via's to form a conformal lining [14]. Additional backside planarization processes are used to remove any diamond growth out of the via. The completed wafer then undergoes standard front side processing. Simulations point to a $3 \mathrm{X}$ increase in output power density $\left(\mathrm{W} / \mathrm{mm}^{2}\right)$ over baseline GaNon-SiC but have yet to be experimentally validated.

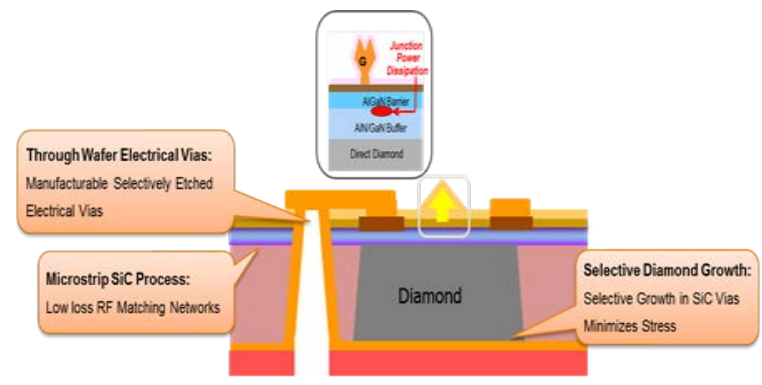

Fig. 5. Diamond filled via GaN HEMT device.

\section{RESULTS}

BAE discrete HEMT devices fabricated using the device first process have achieved the highest microwave output power density reported to date for a GaN-on-Diamond device. Fig. 6 shows constant power $(\mathrm{CW})$ load-pull measurements performed on a $12 \times 50 \mu \mathrm{m}$ GaN-on-Diamond device at $10 \mathrm{GHz}$ produced $11.0 \mathrm{~W} / \mathrm{mm}$ at a $40 \mathrm{~V}$ drain bias with $51 \%$ PAE [10]. This is the first reported GaN-on-Diamond device result utilizing SOA GaN epitaxy from $\mathrm{SiC}$ substrates.

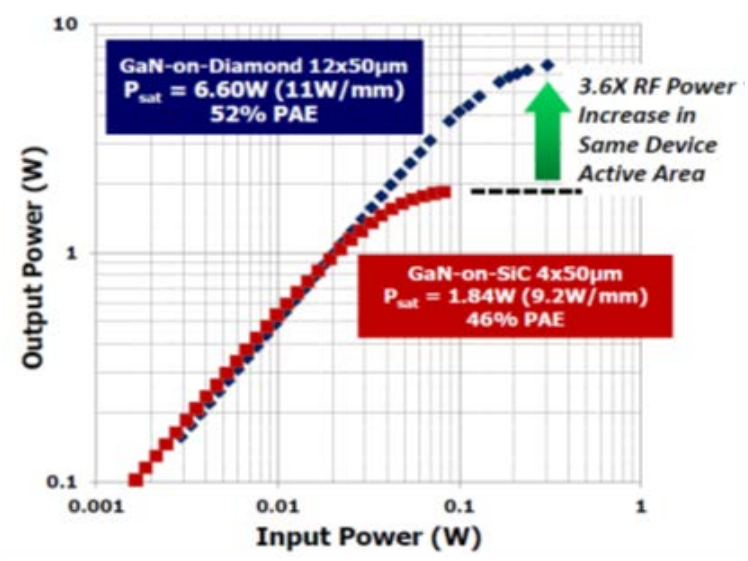

Fig. 6. Comparison of $10 \mathrm{GHz}$ input-output power curves for a GaN-on-SiC and GaN-on-Diamond HEMT [10].

Fig. 7 shows infrared images taken on a $12 \times 50 \mu \mathrm{m}$ GaN-on-Diamond and a $4 \times 50 \mu \mathrm{m}$ GaN-on-SiC HEMT at $20 \mathrm{~W} / \mathrm{mm}$. or comparison with the device. The use of a diamond substrate clearly demonstrates improved heat extraction despite $3 \mathrm{X}$ higher dissipated power within the same active area [10].

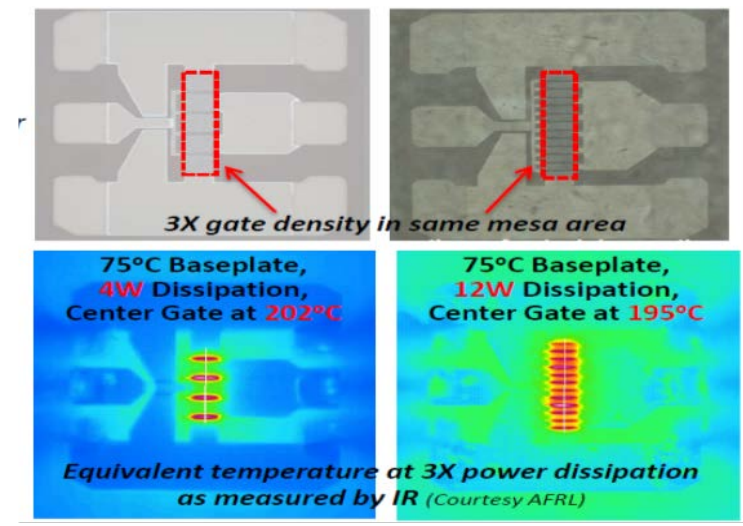

Fig. 7. Comparison of IR images for a $4 \times 50 \mu \mathrm{m} \mathrm{GaN-on-SiC}$ HEMT (left) to that of a $12 \times 50 \mu \mathrm{m}$ GaN-on-Diamond HEMT (right) [10]. 
Both the direct growth and wafer bonding processes require the use of a thin dielectric layer to mechanically bond the diamond substrate to the GaN device layer. The thermal boundary resistance introduced by this interface layer must be minimized to fully exploit diamond's thermal transport properties. Modeling estimates suggest the $\mathrm{GaN}$ Diamond interface resistance remain $<35 \mathrm{~m}^{2} \mathrm{~K} / \mathrm{GW}$ to achieve $>3 X$ 's increase in GaN HEMT areal power density $\left(\mathrm{W} / \mathrm{mm}^{2}\right)$. Fig. 8 shows thermal boundary resistance measurements performed by the University of Bristol using a thermoreflectance technique. Numerous regions on the wafer were explored to create a map that provides insight into the homogeneity of the wafer.

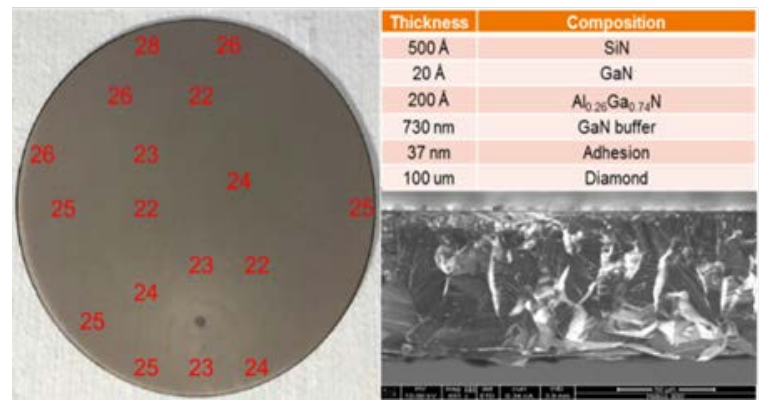

Fig. 8. Thermal boundary resistance $\left(\mathrm{m}^{2} \mathrm{~K} / \mathrm{GW}\right)$ measurements on an Element $675 \mathrm{~mm}$ GaN-on-Diamond wafer.

\section{CONCLUSION}

Future system designs will demand higher levels of electronics integration and miniaturization driving improved thermal management solutions. Initial results of embedding polycrystalline diamond clearly show diamond's improved thermal spreading capability enabling increased areal power density $\left(\mathrm{W} / \mathrm{mm}^{2}\right)$ while maintaining an acceptable junction temperature. While GaN-on-Diamond RF performance has yet to replicate GaN-on-SiC capabilities, this innovative embedded cooling technology shows promise but significant improvements with material quality and producibility are necessary.

\section{ACKNOWLEDGMENT}

The views, opinions, and/or findings contained in this article/presentation are those of the author/presenter and should not be interpreted as representing the official views or policies, either expressed or implied, of the Defense Advanced Research Projects Agency, Air Force or the Department of Defense.

\section{REFERENCES}

[1] J.D. Blevins, History of GaN-on-Diamond Development at Air Force Research Laboratory, Lester Eastman Conference on High Performance Devices 2014.

[2] Y. F. Wu, M. Moore, A. Saxler, T. Wisleder, and P. Parikh, Proc. $64^{\text {th }}$ DRC Tech. Dig., 2006, pp. 151-152.

[3] P.C. Chao, et al., A New High Power GaN-on-Diamond HEMT with Low-Temperature Bonded Substrate Technology, Compound Semiconductor Manufacturing Technology Conference 2013.

[4] G. H. Jessen, et al., "AlGaN/GaN HEMT on Diamond Technology Demonstration", Compound Semiconductor Integrated Circuit Symposium Proceedings, p. 271 -274, 2006.

[5] G.D. Via, et al., "Wafer-scale GaN HEMT performance enhancement by diamond substrate integration", Phys. Status Solidi (2014)

[6] M. Trejo, et al., "Progress towards III-nitrides HEMTs on free-standing diamond substrates for thermal management", Phys. Status Solidi A 208, No. 2, 439-444 (2011).

[7] M. Trejo, et al., "Comparative Study of AlGaN/GaN HEMTs on Free-Standing Diamond and Silicon Substrates for Thermal Effects," Compound Semiconductor Integrated Circuit Symposium 2010.

[8] K. Chabak, et al., "Characterization of First AlGaN/GaN HEMTs on Free-Standing CVD Diamond Substrate," IEEE Electron Device Letters, Vol. 31, No. 2, Feb. 2010.

[9] A. Bar-Cohen, et al., DARPA's Intra/Interchip Enhanced Cooling (ICECool) Program, Compound Semiconductor Manufacturing Technology Conference 2013.

[10] P.C. Chao, et al., GaN-on-Diamond HEMTs with $11 \mathrm{~W} / \mathrm{mm}$ Output Power at 10GHz, MRS 2015.

[11] D. Altman, et al., Development of a Diamond Based Intrachip Cooling Technology for GaN, InterPACK 2015.

[12] D. Altman, et al., "Analysis and Characterization of Thermal Transport in GaN HEMTs on SiC and Diamond Substrates", GOMACTech 2014

[13] D.C. Dumka, P. Saunier, "AlGaN/GaN HEMTs on Diamond Substrate," Proc. 65th DRC Tech. Dig, 2007, pp.31-32.

[14] R. Sandhu, et al., "GaN HEMT Near Junction Heat Removal", CS ManTech Conference 2013.

[15] M. Tyhach, et al., Next Generation Gallium Nitride HEMTs Enabled by Diamond Substrates, Lester Eastman Conference on High Performance Devices 2014. 